\title{
Energy Management for Compressed Air in Cement Industry using Lean Principle
}

\author{
Shunmathi M, Siva M, Arirajan KA, Tamilarasan N
}

\begin{abstract}
Energy is an ultimate source for performing any activities. It is defined as capacity or power to do work, such as the capacity to move an object by the application of force. Energy can exist in a variety of forms such as electrical,mechanica l,chemical,thermal.Energy will not be generated but it can be transformed from one from to another. Energy resources are limited India has approximately $1 \%$ of world energy resources but it has $16 \%$ of world population. The industrial sector comprised 51\% of global delivered energy use in 2007 and grows by an average annual $1.3 \%$ over the projection[worrell Ernst 2004].Approximately half of all industrial energy use is used in specific processes in the energy intensive industries. Compressors are the devices plays major role in every process industries and also a one of the major power consuming area. This paper seeks to identify and eliminate routine wastes which causes higher energy consumption in cement process industry(ULTRA TECH CEMENTS-RDCW) by using suitable layout.
\end{abstract}

Keywords: Compressor, Eliminate,Energy saving, Routine wastes

\section{INTRODUCTION}

Energy is defined as capacity or power to do work, such as the capacity to move an object (of a given mass) by the application of force. Energy can exist in a variety of forms, such as electrical, mechanical, chemical, thermal, or nuclear. Energy will not be generated but it can be transformed from one form to another. Energy management is the means to controlling and reducing your organization's energy consumption. Industry uses more energy than any other end-use sector, consuming about one-half of the worlds. The industrial sector comprised $51 \%$ of global delivered energy use in 2007 and grows by an average annual $1.3 \%$ over the projection. Approximately half of all industrial energy use is used in specific processes in the energy-intensive industries. Controlling and reducing your organization's energy consumption is important because it enables you to

Reduce costs this is becoming increasingly important as energy costs rise.

1. Reduce carbon emissions and the environmental damage that they cause as well as the cost-related implications of carbon taxes and the like, your organization may be keen to reduce its carbon footprint

Revised Manuscript Received on September 08, 2019

Shunmathi M, Research scholar(Ph.D), Mechatronics Engineering, Thiagarajar college of Engineering,Madurai. Shunmathimech@gmail.com

Siva M, PG Scholar, Industrial Engineering, Thiagarajar college of Engineering,Madurai.

Arirajan KA, PG scholar, Manufacturing Engineering, Thiagarajar college of Engineering,Madurai.

Tamilarasan N, PG Scholar, aktamil5@gmail.com, Thiagarajar college of Engineering,Madurai. to promote a green, sustainable image. Recognition as a "green company".

2. Reduce risk - the more energy you consume, the greater the risk that energy price increases or supply shortages could seriously affect your profitability, or even make it impossible you're your business/organization to continue. With energy management you can reduce this risk by reducing your demand for energy and by controlling it so as to make it more predictable.

3. Improves productivity-Energy saving helps to increase the productivity and can provide the products to customers at minimal cost. It will give the competitive advantages for an organization.

4. Improved operational reliability and control

\subsection{PROCESS FLOW DIAGRAM}

Cement Mill

$>$ Compressor Room

Raw Mill

Boiler

$>$ Turbine

$>$ AFR Hopper

$>\mathrm{RM}$ bag filter

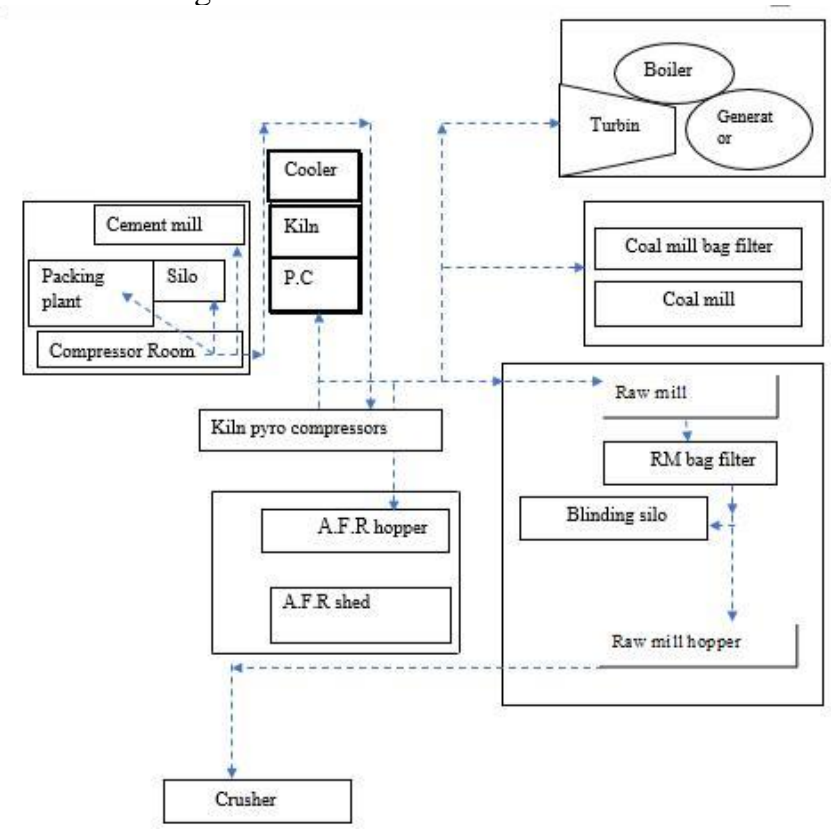

\subsection{DEMAND:}

Energy demand is mainly classified into two types as shown in Fig 1:

1. Non value added process

2. Value added process

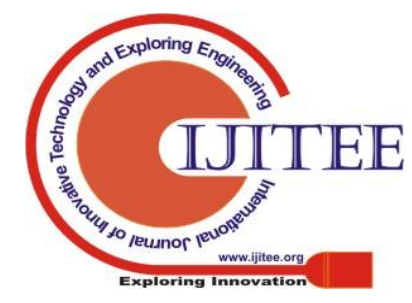




\section{Energy Management for Compressed Air in Cement Industry using Lean Principle}

\section{PROBLEM DESCRIPTION}

\subsection{PROBLEM ENVIRONMENT}

The utilization of compressors is more in all the process industries and also it is one of the major energy consuming components. At the same time efficient and proper utilization of compressors minimizes energy consumption rate.

- Efficient equipment should be maintained at $80 \%$ working condition.

- Compressed air transferred from raw mill hopper to crusher section through conveyor, drainage, air receiver and several number of pipe fittings. It is long distance and vertical pipe line followed to transfer compressed air. which cause pressure drop and air leakage

- Air will be maintained at 6 bar in each and every section but Air have been required only 8 to 10 hours. Remaining hour's air will be supplied to crusher line which has exhausted atmosphere through leakages and valves.It will cause higher inventory, maintenance costs and high level of energy consumption rate.

- A long distance(222meter) has been maintained for to transfer the compressed air

- Flow rate has been reduced from $\left(73 \mathrm{~m} / \mathrm{hr}\right.$. to $\left.64 \mathrm{~m}^{3} / \mathrm{hr}\right)$ due to long distance

- Compressor used in crusher section is a reciprocating typewhich causes maintenance costs and considerable rate (90kwh) of energy consumption. But this compressor "ON" only, when shutdown time.

\subsection{OBJECTIVE}

Reducing the energy consumption $(87 \mathrm{~kW}$ from 232 $\mathrm{kW} /$ day) rate for compressed air supply to crusher section by eliminating the wastes.

\subsection{METHODOLOGY}

To metering the energy consumption and collecting data of the cement plant.To analyse tackle the routine waste for replacing.To develop in six sigma technique and effective way due to analyse the routine waste. It's necessary to eliminate the routine wastes such as transport, inventory, motion, waiting time, over production, over processing, defects (TIMWOOD) for energy efficient solution and savings.PDCA (plan-do-check-act or plan-do-check-adjus t) four step management method used in business for the control and continual improvement of processes and products.It is also known as the deming circle/cycle/wheel, the Stewart cycle, the control circle/cycle, or plan-do-study-act (PDSA). Another version of this PDCA cycle is OPDCA. The added "O" stands for observation or as some versions say: "Observe the current condition." This emphasis on observation and current condition has currency with the literature on lean manufacturing .

\subsection{ENERGY MANAGEMENT SYSTEM:}

The following flow chart explains an energy saving process step by step:

1. Metering your energy consumption and collecting the data.

2. Finding opportunities to save energy, and estimating how much energy each opportunity could save.

3. Taking action to target the opportunities to save energy (i.e.tackling the routine waste and replacing or upgrading the inefficient equipment).

4. Tracking your progress by analyzing your meter data to see how well your energy-saving efforts have worked.

\subsection{ABOUT CEMENT:}

Cement is a material that binds together solid bodies (aggregate) by hardening from a plastic state. Many materials act as adhesives or cement according to this definition. The cement referred to above, which is used for civil engineering and the construction industry, is Portland cement. Portland cement is hydraulic and develops strength primarily by the hydration of the di- and tri-calcium silicates it contains. Hydraulic means that the paste of cement and water will harden under water. Lime, on the other hand, will harden due to the reaction with carbon dioxide from the air

\subsection{VARITIES \& GRADE OF CEMENTS:}

India is marketing several varieties of cement which include Ordinary Portland Cement (OPC), Portland Pozallona Cement (PPC), Portland Blast Furnace Slag Cement (PBFS), Rapid Setting Cement, Oil Well Cement, White Cement etc.,

\subsubsection{DEFINITIONS:}

\section{Cement:}

Cement is a mixture of cement clinker and gypsum ground together in to a powder.

\section{Clinker:}

The stony residue from burnt coal or from a furnace.

\section{Gypsum:}

Calcium sulfate is used to regulate settling time.

\section{Fly Ash:}

Ash produced in small dark flecks by the burning of powdered coal or other materials and carried into the air.

\section{DESIGN CONSIDERATION}

\subsection{DESIGN CONSIDERATION OF METERING THE ENERGY CONSUMPTION AND COLLECTING DATA}

Table 1 Compressor type and their details:

\begin{tabular}{|c|c|c|c|c|c|c|c|}
\hline $\begin{array}{c}\text { Sn } \\
0\end{array}$ & $\begin{array}{l}\text { Equipment } \\
\text { ID }\end{array}$ & Type & $\begin{array}{l}\text { Capacity } \\
(\mathrm{m} 3 / \mathrm{hr})\end{array}$ & $\begin{array}{l}C F \\
M\end{array}$ & $\begin{array}{l}\text { Motor } \\
\text { power } \\
(\mathrm{Kw})\end{array}$ & $\begin{array}{c}\text { Nax } \\
\text { pressure } \\
(\mathrm{kg} / \mathrm{cm} 2)\end{array}$ & Operation \\
\hline 1 & D11CP1 & Screw & 1500 & 909 & 132 & 75 & Yes \\
\hline 2 & D11CP2 & Screw & 1500 & 909 & 132 & 75 & Standby \\
\hline 3 & D11CP3 & Screw & 1500 & 909 & 132 & 75 & Standby \\
\hline 4 & D11CP4 & Screw & 1500 & 909 & 132 & 75 & Standby \\
\hline 5 & D21CP1 & Screw & 1000 & 606 & 90 & 75 & Yes \\
\hline 6 & D21CP3 & Screw & 1000 & 606 & 90 & 75 & Yes \\
\hline 7 & TS03120HN & Reciprocating & 19 & 11 & 2.5 & $\begin{array}{c}12 \\
\mathrm{kgf} / \mathrm{cm} 2\end{array}$ & Standby \\
\hline 8 & SS07090HN & Reciprocating & 55 & 33 & 8 & $\stackrel{9}{9}$ & Standby \\
\hline & $\begin{array}{l}\text { Publishec } \\
\text { Blue Eye } \\
\text { \& Scienc }\end{array}$ & $\begin{array}{l}\text { telligence } \\
\text { ublicatio }\end{array}$ & & & & & \\
\hline
\end{tabular}


Table 2 No of hour Compressed air used in crusher section:

\begin{tabular}{|c|c|c|c|c|}
\hline Operation & $\begin{array}{c}\text { Flow } \\
(72.23 \mathrm{~m} 3 / \mathrm{hr} .)\end{array}$ & C.F.M & $\begin{array}{c}\text { Power } \\
(\mathrm{Kw})\end{array}$ & Cost/year \\
\hline $\begin{array}{c}9 \mathrm{hr} . \\
\text { operation }\end{array}$ & 650.07 & 396 & 87.10 & $129,357.420$ \\
\hline $\begin{array}{c}24 \mathrm{hr} . \\
\text { operation }\end{array}$ & 1733.52 & 1057 & 232.29 & $344,950.65$ \\
& & & & \\
\hline
\end{tabular}

- $1 \mathrm{~m} 3 / \mathrm{hr}=0.134 \mathrm{Kw}$

- 1 C.F.M = $1.64 \mathrm{~m} 3 / \mathrm{hr}$.

- 1 C.F.M $=28.31 \mathrm{lpm}$

Table 3 Compressed air transferred through this component:

\begin{tabular}{|c|c|c|}
\hline S.No & Materials & Quantity \\
\hline 1 & Elbows & 20 \\
\hline 2 & G.I pipes & $38(6$ meter $* 1$ inch $)$ \\
\hline 3 & Air receiver & $7(100$ liter $)$ \\
\hline 4 & Air blaster & 1 \\
\hline 5 & Bag filter & 1 \\
\hline 6 & Pressure Shut valve & \multicolumn{2}{|c|}{} \\
\hline
\end{tabular}

Power consumption $=\left(72.23 \mathrm{~m}^{3} / \mathrm{hr} . * 0.134 * 15 * 330\right)$

$$
\begin{aligned}
= & 47910 \mathrm{Kwh} / \text { day } \\
& =47910 * 4.5 \\
= & 215,595 \mathrm{Rs} / \text { annum }
\end{aligned}
$$

\section{Note:}

$\mathrm{Q}=72.23 \mathrm{~m}^{3} / \mathrm{hr}$.

$1 \mathrm{~m}^{3} / \mathrm{hr}=0.314 \mathrm{Kw}$

Running hr= 24hr.

Working days $=330$

Pressure drop will be occurs at,

$>$ Vertical line and Horizontal line

$>$ Pipe joining places

$>$ Valve and bends(include elbow, L-bend)

\section{FORMULAE USED:}

- Air density $=\mathrm{P} /(\mathrm{R} * \mathrm{~T})$

$$
=101.325 /(287.058 * 304
$$

Air density $=1.167 \mathrm{~kg} / \mathrm{m}^{3}$
- Velocity of air flow through a circular pipe,

Velocity $=(4 *$ flow rate $) /\left(л * D^{2}\right)$

$$
=(4 * 0.02006) /\left(\Omega * 0.025^{2}\right)
$$

$$
\text { Velocity }=41 \mathrm{~m} / \mathrm{s}
$$

- Reynolds number = (density*flow velocity*diameter)/(absolute viscosity)

$$
\text { Air density }=1.2 \mathrm{~kg} / \mathrm{m}^{3}
$$

Kinematic viscosity $=1.6 \times 10^{\wedge} 5 \mathrm{~kg} / \mathrm{m}^{3}$

$=\left(1.2 * 41^{2 *} 0.025\right) /\left(1.98 \times 10^{\wedge} 5\right)$

$\operatorname{Re}=62121.21$ (turbulent flow)

- Friction factor for turbulent flow from pipe friction chart

$$
\mathrm{f}=0.033
$$

- Reynolds number = (density*flow velocity*diameter $) /($ absolute viscosity)

$$
\text { Air density }=1.2 \mathrm{~kg} / \mathrm{m}^{3}
$$

Kinematic viscosity $=1.6 \times 10^{5} \mathrm{~kg} / \mathrm{m}^{3}$

$=(1.2 * 41 * 0.025) /\left(1.98 \times 10^{-} 5\right)$

$$
\mathrm{Re}=63674 \text { (turbulent }
$$

flow)

$$
\begin{array}{r}
=\left(1.2^{*} 41^{2 *} 0.025\right) /\left(1.98 \times 10^{\wedge} 5\right) \\
\operatorname{Re} \quad=\quad 62121.21
\end{array}
$$

(turbulent flow)

- Friction head $\mathrm{Hf}=\left(4 * \mathrm{f} * \mathrm{~L} * \mathrm{v}^{2} / 2 * \mathrm{~g} * \mathrm{~d}\right)$

$$
\begin{aligned}
& =\left(4 * 0.033 * 222 * 41^{2}\right) /(2 * 9.81 * 0.025) \\
& \text { Hf }=100,428
\end{aligned}
$$

- Pressure drop in $\Delta \mathrm{P}=\left((\mathrm{f}) *(1 / \mathrm{d}) *(\mathrm{e} / 2)^{*}\left(\mathrm{v}^{2}\right)\right)$

$$
\begin{aligned}
& =\left((0.033) *(222 / 0.025) *(1.23 / 2)^{*}\left(41^{2}\right)\right) \\
& =3.39 \times 10^{\wedge} 5 \\
& =3.3 \text { bar (approximately) } \\
& \text { Note, } \\
& \qquad \begin{aligned}
\mathrm{f} & =\text { friction factor } \\
\mathrm{d} & =\text { diameter of pipe } \\
\mathrm{e} & =\text { compressed air density } \\
\mathrm{v} & =\text { flow velocity }
\end{aligned}
\end{aligned}
$$




\section{Energy Management for Compressed Air in Cement Industry using Lean Principle}

\section{PUMP UP TEST, ( compressor ID D11CP2)}

$\mathrm{P} 1$ (initial pressure) $=0 \mathrm{bar}$

$\mathrm{P} 2($ final pressure $)=6 \mathrm{bar}$

P0 $($ atmospheric pressure $)=1.026$ bar

$(\mathrm{P} 2-\mathrm{P} 1 / \mathrm{P} 0)=5.85 \mathrm{bar}$

$\mathrm{T} 1=34^{\circ} \mathrm{C}$

$\mathrm{T} 2=36^{\circ} \mathrm{C}$

Temp correct $=(273+\mathrm{t} 1 / 273+\mathrm{t} 2)$

$$
=99.35
$$

Pump up time,

$$
\begin{aligned}
1-2 \mathrm{bar} & =24.56 \mathrm{sec} \\
2-3 \mathrm{bar} & =24.6 \mathrm{sec} \\
3-4 \mathrm{bar} & =25.06 \mathrm{sec} \\
4-5 \mathrm{bar}=26.7 \mathrm{sec} & \\
5-6 \mathrm{bar}= & 26.76 \mathrm{sec} \\
\text { Total } & =2.58 \text { minutes. Avg }
\end{aligned}
$$

Actual free air discharge $(\mathrm{Q})=(\mathrm{P} 2-\mathrm{P} 1) / \mathrm{P} 0 *(\mathrm{~V} / \mathrm{T})$

$$
\begin{aligned}
= & (6-0) / 1.026 *(8.89751 / 2.58) \\
& =20.107 \mathrm{~m}^{3} / \text { minutes } \\
& =1203.93 \mathrm{~m}^{3} / \mathrm{hr} .
\end{aligned}
$$

Compressor efficiency $(\eta ́)=$ Actual free air discharge $(Q)$ / capacity of compressor (C)

$$
\begin{aligned}
& =1203.93 / 1500 \\
& =80.26 \%
\end{aligned}
$$

Table 5Compressor efficiency through pump up test:

\begin{tabular}{|c|c|c|c|}
\hline $\begin{array}{c}\text { Compressor } \\
\text { ID }\end{array}$ & $\begin{array}{c}\text { FAD } \\
\left(\mathbf{m}^{3} / \mathbf{h r}\right)\end{array}$ & $\begin{array}{c}\text { Capacity } \\
\left(\mathbf{m}^{3} / \mathbf{h r} .\right)\end{array}$ & $\begin{array}{c}\text { Compressor } \\
(\% \mathbf{\eta})\end{array}$ \\
\hline D11CP1 & 1347.16 & 1500 & 89.81 \\
\hline D11CP3 & 1343.40 & 1500 & 89.56 \\
\hline D11CP4 & 1337.71 & 1500 & 89.18 \\
\hline D21CP1 & 867.65 & 1000 & 86.75 \\
\hline D21CP3 & 876.04 & 1000 & 87.60 \\
\hline
\end{tabular}

Actual flow to normal flow $=($ Flow rate $*$ pressure in bar *273)/ $\left(273\right.$ + outlet temperature in Deg $\left.{ }^{\circ} \mathrm{C}\right)$

$$
\begin{aligned}
& =(72.23 * 6 * 273) /(273+36) \\
& =382.59
\end{aligned}
$$

Table 6 Standard values for Pressure drop and Power loss depending upon "NB":

\begin{tabular}{|c|c|c|c|}
\hline S.No & $\begin{array}{c}\text { "NB" nominal } \\
\text { bore (mm) }\end{array}$ & $\begin{array}{c}\text { Pressure drop per } \\
100 \mathrm{~m} \text { (Bar) }\end{array}$ & $\begin{array}{l}\text { Power loss } \\
(\mathrm{Kw})\end{array}$ \\
\hline 1 & 40 & 1.80 & 9.5 \\
\hline 2 & 50 & 0.65 & 3.4 \\
\hline 3 & 65 & 0.22 & 1.2 \\
\hline 4 & 80 & 0.04 & 0.2 \\
\hline 5 & 100 & 0.02 & 0.1 \\
\hline
\end{tabular}

\subsection{APPLICATION OF SUITABLE TECHNIQUE TO PROVIDE ENERGY EFFICIENT SOLUTION}

The plan for energy saving were developed based upon process impact, cost and associated risks. Thus a solution cost / benefit analysis was performed, for an organization. An analysis work had done in this phase. The brain storming section had conducted under the guide lines of senior engineer and plant engineer mechanical department with participant of 3 person from mechanical department, 4 person from CCR (central control room), and 3person from crusher section. Finally the following solution had developed from this session.

\section{PROPOSED WORK}

Through brainstorming following ideas are generated to optimize the compressor

Option-1

(i) Compressed air supply from A.F.H to crusher section

(ii) Compressed air supply from Raw Mill Hopper to Crusher section

\section{Option- 2}

(i) Separately Compressor Placed in Crusher Section

I. Compressed air supply from Raw Mill Hopper to Crushersection

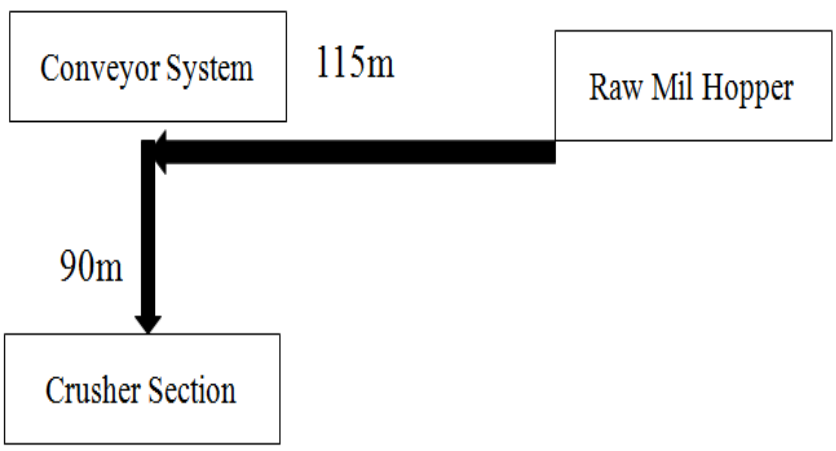


Table1 Bill of material and other expenses for 1inch pipe fittings:

\begin{tabular}{|c|c|c|}
\hline $\begin{array}{l}\text { Type of Pipe } \\
\text { fittings }\end{array}$ & Quantity & Cost \\
\hline G.I pipes & 19 & 17,100 \\
\hline $\begin{array}{c}\text { Automatic } \\
\text { Pressure shut } \\
\text { valve }\end{array}$ & 1 & 4500 (approximately) \\
\hline $\begin{array}{c}\text { Flow meter } \\
\text { setup }\end{array}$ & 1 & 9500 \\
\hline $90^{\circ}$ Long bend & 8 & 184 \\
\hline Teflon tape & 7 & 105 \\
\hline \multicolumn{2}{|c|}{ Direct material cost } & 31389 \\
\hline $\begin{array}{l}\text { Direct labour } \\
\text { cost }\end{array}$ & $\begin{array}{l}3 \text { person } x \\
2 \text { days } x \\
500 R \text { s/day }\end{array}$ & 3000 \\
\hline $\begin{array}{l}\text { Other } \\
\text { expenses }\end{array}$ & $\begin{array}{l}\text { Food, } \\
\text { electricity cost }\end{array}$ & 1000 \\
\hline Total cost & & 35,389 \\
\hline
\end{tabular}

\subsection{Observation:}

- $\quad$ Requirement of compressed air should be 38 CFM. but supplying the $46 \mathrm{CFM}$ of compressed air to crusher section

- Distance will have maintain 205meter

- Automatic pressure shut valve required to open and close the compressed air flow line.

- $\quad$ 1inch NB nominal bore (dia) used at presently. The pipe NB increases to $11 / 4$ inch, by which pressure drop will be decreased ( 0.65 bar) than 1 " inch pipe (2.79 bar)

- $\quad$ Pressure drop in (bar) $\Delta \mathrm{P}=\left((\mathrm{f}) *(1 / \mathrm{d}) *(\mathrm{e} / 2)^{*}\left(\mathrm{v}^{2}\right)\right)$

(1 inch pipe)

$$
\begin{aligned}
&=\left((0.033) *(205 / 0.025) *(1.23 / 2) *\left(41^{2}\right)\right) \\
&= 2.79 \times 10^{\wedge} 5 \\
&=\mathbf{2 . 7 9} \text { bar }
\end{aligned}
$$

Incase change NB size from 1inch to $11 / 4$ inch, pressure drop will be reduced

- $\quad$ Pressure drop in (bar) $\Delta \mathrm{P}=\left((\mathrm{f}) *(1 / \mathrm{d}) *(\mathrm{e} / 2) *\left(\mathrm{v}^{2}\right)\right)$

$$
\text { (1.1/4 inch pipe) }
$$

$$
\begin{aligned}
=((0.027) *(205 / 0.032) *(1.23 / 2) & \left.*\left(24.75^{2}\right)\right) \\
= & 0.6516 \times 10^{\wedge} 5 \\
& =\mathbf{0 . 6 5} \text { bar }
\end{aligned}
$$

Expected Benefits:

- Maintenance cost of pipe line will be reduced

- Power saving in crusher section $145 \mathrm{Kw}$

\begin{tabular}{|c|c|c|}
\hline $\begin{array}{l}\text { Type of Pipe } \\
\text { fittings }\end{array}$ & $0 \ldots$ & Cost \\
\hline G.I pipes & 15 (90meter) & 13,500 \\
\hline $\begin{array}{c}\text { Automatic } \\
\text { Pressure shut } \\
\text { valve }\end{array}$ & 1 & 4500(approximately) \\
\hline Flow meter setup & 1 & 9500 \\
\hline $90^{8}$ Long bend & 7 & 161 \\
\hline Teflon tape & 5 & 75 \\
\hline \multicolumn{2}{|c|}{ Direct material cost } & 27,736 \\
\hline $\begin{array}{l}\text { Direct labour } \\
\text { cost }\end{array}$ & $\begin{array}{l}3 \text { person } x \\
2 \text { days } x \\
500 \text { Rs/day }\end{array}$ & 3000 \\
\hline Other expenses & $\begin{array}{l}\text { Food, electricity } \\
\text { cost }\end{array}$ & 1000 \\
\hline Total cost & & 31,736 \\
\hline
\end{tabular}

Expected cost savings:

$$
\begin{aligned}
& =\left(126 \mathrm{Kw}^{*} 15 \mathrm{hr} * 330 \mathrm{days} * 4.5 \mathrm{Rs} / \mathrm{unit}\right) \\
& =215,399 \mathrm{Rs} / \text { annum }
\end{aligned}
$$

Payback period $=$ initial investment/annual cash flow

$$
\begin{aligned}
& =35,389 / 215,399 \\
& =2 \text { month }
\end{aligned}
$$

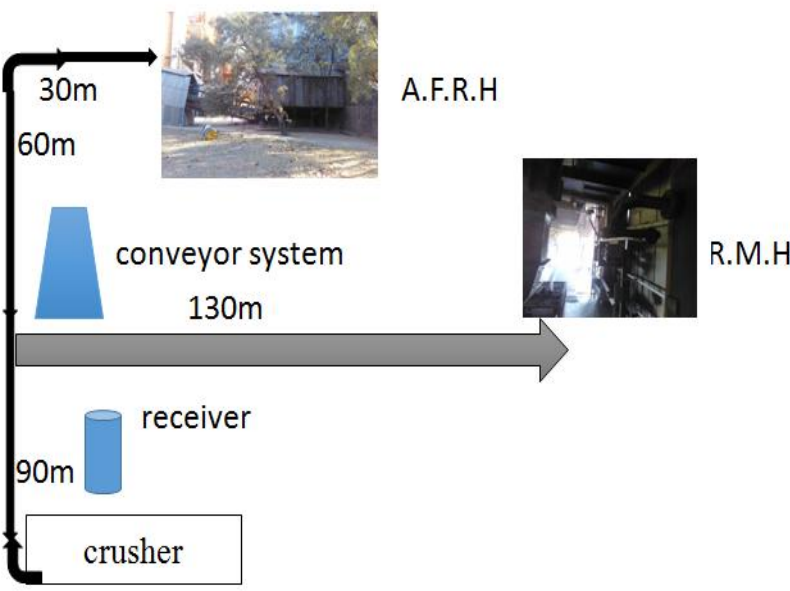

Table 2 Bill of material of pipe fittings and other cost:

Proposal : (under option-1)

Separately new Compressor will be placed in Crusher Section

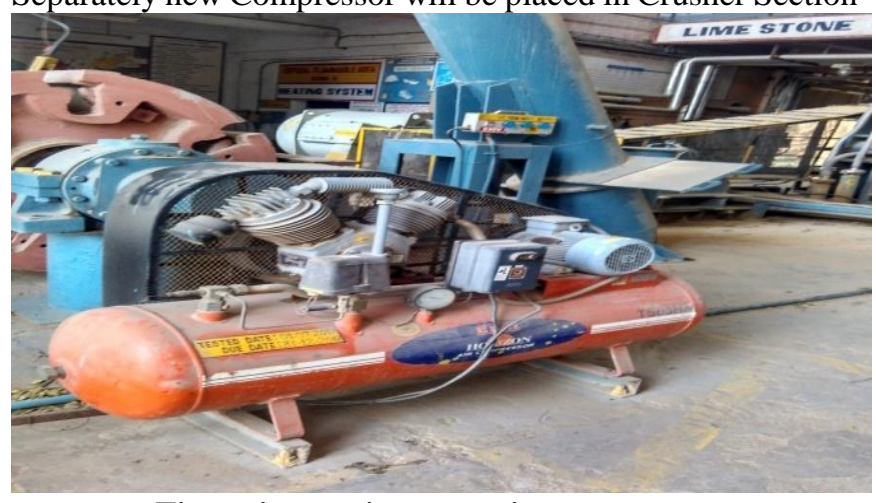

Fig reciprocating type air compressor

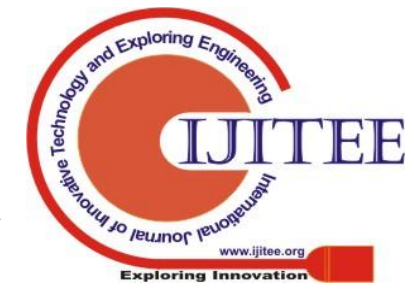




\section{Energy Management for Compressed Air in Cement Industry using Lean Principle}

\subsection{THEORETICAL ANALYSIS:}

- Requirement of compressed air should be 38 CFM per hr. but supplying the $46 \mathrm{CFM}$ of compressed air to crusher section

- Distance will have maintain $180 \mathrm{~m}$

- $\quad$ Pressure drop in (bar) $\Delta \mathrm{P}=\left((\mathrm{f}) *(1 / \mathrm{d}) *(\mathrm{e} / 2)^{*}\left(\mathrm{v}^{2}\right)\right)$ (1 inch pipe)

$$
\begin{aligned}
=((0.033) *(180 / 0.025) *(1.23 / 2) & \left.*\left(41^{2}\right)\right) \\
& =2.45 \times 10^{\wedge} 5 \\
& =\mathbf{2 . 4 5} \text { bar }
\end{aligned}
$$

Incase change NB size from 1inch to $11 / 4$ inch, pressure drop will be reduced

- $\quad$ Pressure drop in (bar) $\Delta \mathrm{P}=\left((\mathrm{f}) *(1 / \mathrm{d})^{*}(\mathrm{e} / 2)^{*}\left(\mathrm{v}^{2}\right)\right)$

(1.1/4 inch pipe)

$$
\begin{aligned}
&=\left((0.027) *(180 / 0.032) *(1.23 / 2) *\left(24.75^{2}\right)\right) \\
&=0.57 \times 10^{\wedge} 5 \\
&=\mathbf{0 . 5 7} \mathbf{~ b a r}
\end{aligned}
$$

Payback period =initial investment/annual cash flow

$$
\begin{aligned}
& =31,736 / 215,399 \\
& =1 \text { month }
\end{aligned}
$$

- Automatic pressure shut valve required to open and close the compressed air flow line.

- $\quad$ NB nominal bore $25 \mathrm{~mm}$ (dia) used at presently. The pipe NB increases to $1 \frac{1}{4}$ " inch from 1 " inch, by which pressure drop will be decrease from 2.45 bar to 0.57 bar.

\section{- Expected Benefits:}

- Maintenance cost of pipe line will be reduced

- Power saving in crusher section $145 \mathrm{Kw}$

\section{Expected cost saving $(126 \mathrm{Kw} * 15 \mathrm{hr} * 330 \mathrm{days} * 4.5 \mathrm{Rs} / \mathrm{unit})$}

\section{$=2,15,399 \mathrm{Rs} /$ annum}

Payback period $=$ initial investment/ annul cash flow

$$
\begin{aligned}
& =172,145 / 2,15,399 \\
& =8 \text { month }
\end{aligned}
$$

\section{CHECK:}

Study the actual results (measured and collected in "DO" above) and compare against the expected results (targets or goals from the "PLAN") to ascertain any differences. Look for deviation in implementation from the plan and also look for the appropriateness and completeness of the plan to enable the execution, i.e., "Do". Charting data can make this much easier to see trends over several PDCA cycles and in order to convert the collected data into information. Information is what you need for the next step "ACT"

\section{ACT:}

This step says that If the change did not work, go through the cycle again with a different plan. If you were successful, incorporate what you learned from the test into wider changes. Use what you learned to plan new improvements, beginning the cycle again. In our case, plan has been worked successfully with an expected energy saving of $145 \mathrm{KW} /$ day and the results for savings in with and without replacement were tabulated below. If an organization requires additional energy saving, then have to make the new plans and repeat the cycles if possible.

\section{CONCLUSION}

To implementing the plan 2 an expected energy saving of 0.29 bar pressure and $10.21 \mathrm{KW}$ can be achieved. To implement the new plan and maintenance activities and air leaks will be minimized and also distance will be minimized. In this plan2,proposed layout automatic control system and essential component like bends, gate valve and flow meter. Using of the compressed air from long distance which create high pressure drop,volume air losses.

\section{REFERENCES}

1. Gustav Nyren, 2007. Product Development According to Six-Sigms and DMAIC Improvement Cycle. Master Degree in Quality Management. Lulea University of Technology.

2. Peter M. O'Rourke, 2005. A Multiple-Case Analysis of Lean Sigma Deployment and Implementation Strategies. Master Thesis. Force Inst Of Tech Wright-Patterson AFB Oh School Of Engineering And Management.

3. Antony J., Kumar M., Labib A.: A gearing Six Sigma into UK manufacturing SME's: results from a pilot study. J. Oper. Res. Soc. 4, 482-493 (2008)

4. Arnheiter E.D., Maleyeff J.: The integration of Lean Management and Six Sigma. TQM Mag. 17, 5-18 (2005)

5. Aslan D., Demir S.: Laboratuar Tibbında Alt1 Sigma Kalite Yönetimi. Türk Biyokimya Dergisi 30, 272-278 (2005)

6. Atmaca E., Girenes S.S.: Literature survey: Lean Six Sigma methodology. J. Fac. Eng. Archit. Gazi Univ. 24(4), 605-612 (2009)

7. Banuelas R., Antony J., Brace M.: An application of Six Sigma to reduce waste. Qual. Reliab. Eng. Int. 25, 553-570 (2005)

8. Bendell T.: Structuring business process improvement methodologies. Total Qual. Manag. 16(8-9), 969-978 (2005)

9. Blakeslee, Jr., J.A.: Implementing the Six Sigma solution. Qual. Prog. 32, 77-85 (1999)

10. Bossert J., Walker R.: Total quality management and performance, an evaluation of the evidence and lessons for research on public organizations. Public Perform. Manag. Rev. J. 26, 111-131 (2002)

11. Brett C., Queen P.: Streamlining enterprise records managements with Lean Six Sigma. Inf. Manag. J. 39, 58-62 (2005)

12. D'Angelo R., Zarbo R.J.:The Henry Ford production system: measures of process defects and waste in surgical pathology as a basis for quality improvement initiatives. Am. J. Clin. Pathol. 3,423-429 (2007)

13. De Mast J., Schippers W.A.J., Does R.J.M.M., Van Den Heuvel E.R.: Steps and strategies in process improvement. Qual. Reliab. Eng. Int. 16, 301-311 (2000)

14. Devane T.: Integrating Lean Six Sigma and High-Performance Organizations, pp. 5-123. 1st edn. Pfeiffer, San Francisco (2004)

15. Dubai Quality Group: The Birth of Lean Sigma, vol. 3, pp. 11-38 The Manage Mentor, Dubai (2003)

\section{AUTHORS PROFILE}

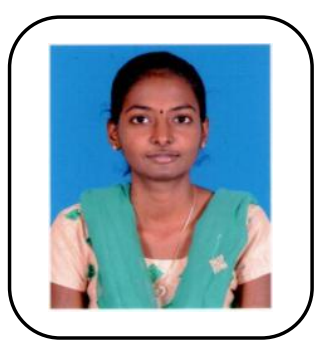

I'm Shunmathi;A young and dynamic Mechatronics Engineer. She received the ME degree in Mechatronics Engineering from the Thiagarajar college of Engineering in 2018 at Madurai.I am currently working $\mathrm{PhD}$ in Mechanical Engineering.Area of interest in control system,robotics working in these fields.My crave towards arts and programming made me choose this discipline of engineering. In myself entrained in seeking to learn new technologies and recent advancement in Robotics.I also work to mathematical modelling of power electronics drives and control. 